\title{
Assessment Of Maternity Staff Training And Knowledge Of Obstetric Care In Burkina Faso: A Repeated Cross-Sectional Study
}

This article was published in the following Dove Press journal: International Journal of Women's Health

\author{
Adama Baguiya (D)' \\ Ivlabèhiré Bertrand Meda ${ }^{1}{ }^{\prime}$ \\ Abou Coulibaly (1D' \\ Mahamadou Fayama ${ }^{2}$ \\ Djénéba Sanon Ouédraogo $\mathbb{D}^{3}$ \\ Souleymane $\mathrm{Zan}^{4}$ \\ Seydou Bélemviré ${ }^{3}$ \\ Henri Gautier Ouédraogo (D) \\ Séni Kouanda $\mathbb{D}^{1,5}$ \\ 'Research Institute of Health Sciences \\ (IRSS), Ouagadougou, Burkina Faso; \\ ${ }^{2}$ Ministry of Health, Ouagadougou, \\ Burkina Faso; ${ }^{3}$ United Nations Fund for \\ Papulation (UNFPA), Ouagadougou, \\ Burkina Faso; ${ }^{4}$ World Health \\ Organization, Cotonou, Benin; ${ }^{5}$ African \\ Institute of Public Health, Ouagadougou, \\ Burkina Faso
}

Correspondence: Adama Baguiya

Research Institute of Health Sciences (IRSS), 06 P.O. Box 9889, Ouagadougou 06, Burkina Faso

Tel +22670878370

Email abaguiya@gmail.com
Background and aim: The quality of maternity care in low-income countries has often been questioned. The objective of this study was to describe the trend of the percentage of staff trained on selected obstetric care topics and their level of knowledge of maternal care over a 5-year period in Burkina Faso.

Methods: We conducted a secondary analysis of data from two national emergency obstetric and newborn care (EmONC) needs assessments. Staff members' knowledge scores were determined at the facility level for 2010 and 2014 and were further categorized into low (less than $50 \%$ ), medium (50 to $74 \%$ ) or high (at least $75 \%$ ) levels. We used McNemar's test with a 5\% significance level to compare the distribution of the proportions in 2010 versus 2014 . Results: Out of 789 facilities surveyed in the 2014 assessment, 736 (93.3\%) were eligible for this study. Most of them were primary healthcare centers (87.2\%). Overall, $21.6 \%$ $(n=197)$ of health workers in 2010 and $39 \%$ in 2014 were midwives. The proportions of staff who received training on focused antenatal care (FANC) and on how to perform active management of the third stage of labor (AMSTL) have increased by $15.8 \%$ and $14.7 \%$, respectively. A significant proportion of facilities had health workers with a low level of knowledge of FANC $(p<0.001)$, the parameters that indicate the start of labor $(p<0.001)$, the monitoring of labor progress $(p<0.001)$ and AMSTL $(p<0.001)$. There was no significant change in staff knowledge in hospitals over the 5-year period.

Conclusion: From 2010 to 2014, the proportion of staff trained in obstetric care has increased. Their level of knowledge also improved, except in hospitals. However, further efforts are needed to reach a high level of knowledge.

Keywords: obstetric care, Burkina Faso, knowledge, maternity

\section{Background}

An estimated 303,000 women die every year during pregnancy, childbirth or in the postpartum period. ${ }^{1}$ Through the Millennium Development Goals (MDGs) 4 and 5 and the Sustainable Development Goals (SDGs), ${ }^{2}$ policymakers, program managers and the United Nations (UN) agencies have committed themselves to mitigate this high level of maternal and neonatal mortality. Tremendous progress was made during the era of the MDGs, but unfinished business still remains, as many countries did not reach their targets, and their quality of care has yet to improve. ${ }^{3,4}$

The skilled birth attendance rate has significantly increased in many subSaharan African countries, including Burkina Faso. ${ }^{5}$ More women are attending health facilities, and more patients with complications are now gaining access to 
hospitals. Consequently, a higher proportion of women with potential complications that could have led to maternal or perinatal death have better access to services. ${ }^{6}$ These circumstances provide a great opportunity to prevent avoidable maternal and neonatal deaths. However, to achieve this goal, facilities should be able to deliver highquality services before, during and after childbirth. ${ }^{6,7}$

The quality of maternity care in low-income countries has often been questioned and is reported to be worst for the poorest individuals. ${ }^{8-10}$ Many interventions have increased service utilization but have failed to reduce maternal and newborn mortality ${ }^{11}$ because of challenges in the healthcare provision environment and poor staff knowledge and skills. ${ }^{9,12,13}$ In a systematic review by Kyei-Nimakoh and colleagues, on the barriers to access to obstetric care in sub-Saharan Africa, poor staff knowledge and skills and inadequate preservice and in-service training, among other factors, were reported as potential barriers and challenges that deter health facilities' utilization and worsen women's experience in maternity wards. ${ }^{14}$

Almost three-quarters of maternal deaths are due to direct obstetric complications. ${ }^{15}$ Therefore, childbirth and the immediate postpartum period are critical; thus, the level of preparedness of maternity staff to deliver highquality care for maternal conditions during that period, particularly their skills and knowledge on emergency obstetric and newborn care (EmONC), are key in averting maternal and neonatal deaths. ${ }^{16}$

Burkina Faso is a low-income country located in West Africa, with a population of $17,880,386$ inhabitants in 2014 and a high maternal mortality ratio (341 maternal deaths per 100,000 live births in 2015). ${ }^{17}$ From 2010 to 2014, a myriad of complex interventions have been implemented across the country to improve the demand and delivery of maternal and child health services, including the following: national-level programs such as the Partnership for Maternal, Neonatal and Child Health $(\mathrm{PMNCH}),{ }^{18}$ a subsidy of EmONC; ${ }^{19}$ subnational-level programs such as $\mathrm{H} 4+$, funded by the Canadian International Development Agency (CIDA) in the northern and north-central regions; ${ }^{20}$ and performance-based financing (PBF). ${ }^{21}$ These programs included capacity-strengthening components, but there is no evidence on how they have impacted staff knowledge on obstetric care over time. Some studies have evaluated changes in knowledge after training that lasted 6 to 12 months in other countries. ${ }^{22}$ However, there still is a gap in how this knowledge evolves over time in real-life environments. In this observational study, we assessed the trend in staff knowledge on selected obstetric care topics in Burkina Faso over a 5-year period. The Burkina Faso 2010 EmONC needs assessment reported low availability and quality of EmONC services. Five years later, no significant improvement in these areas has been detected. ${ }^{23}$ Therefore, we sought to understand how staff profiles and knowledge of obstetric care has changed over that time period. This information can help decision makers in planning capacity building strategies and monitor staff knowledge on key services in Burkina Faso and other countries. Thus, the objective of this study was to use national EmONC needs assessment data to compare the proportion of staff trained on selected obstetric care topics and their level of knowledge of maternal care in 2010 versus in 2014.

\section{Methods \\ Study Design And Setting}

We conducted a secondary data analysis of repeated crosssectional surveys carried out in Burkina Faso in 2010 and 2014 for the EmONC needs assessment.

As of 2014, Burkina Faso had 1678 health centers (HC) and 60 public hospitals in a 3-tier healthcare system across 13 regions. ${ }^{24}$ The country health workforce is characterized by a shortage of medical staff. The majority of staff members in maternity wards are nurses, midwives and assistant midwives. $^{25}$ Due to the shortage of midwives, the Government annually recruits females with primary or secondary school levels and trains them for two years on basic maternity services, such as antenatal care and uncomplicated deliveries, and on how to identify complicated cases for referral to a higher level of care. In this study, this category of staff is referred to as "assistant midwives". They are usually deployed in health centers of rural and remote areas where there are no (or not enough) midwives. In some facilities of urban areas, they work under the supervision of midwives.

After the 2010 EmONC needs assessment, many activities were carried out to improve EmONC services delivery and quality. These activities were led by the Family Health Directorate (DSF) of the Ministry of Health, in partnership with the World Health Organization (WHO), the United Nations Children Fund (UNICEF) and the United Nations Fund for Population (UNFPA). These activities included upgrading the equipment and other aspects of the health centers (appointment of a general practitioner, equipment of biomedical laboratories and training of staff in the performance of all EmONC signal functions). 


\section{Data Source}

This study used secondary data from Burkina Faso 2010 and 2014 EmONC needs assessments. During those surveys, health workers' answers to questions on specific topics were captured using a standardized questionnaire, which is module 7 of the EmONC needs assessment tools, developed by the UN agencies (WHO, UNFPA and UNICEF) in collaboration with Averting Maternal Deaths and Disabilities (AMDD). Information on the methodology and the results of the assessments can be found elsewhere. $^{23,26}$

Module 7 is a checklist for assessing the knowledge of maternity care providers of maternal and neonatal care. The questionnaire was adapted by the national assessment steering committee in 2010 and used in 2014 as well. It was pretested before the assessment in 2010 and during each round of the field workers' training session.

Investigators trained data collectors to conduct face-toface confidential interviews with staff members one at a time without any interference. The interviewers asked each question and waited for the interviewees' spontaneous answers; the interviewers then confirmed that the interviewees had nothing else to add, until they declared that they were done. The interviewers would circle 1 for mentioned items; otherwise, they would circle 0 and move to the next question. Data collection followed the same process in both surveys.

\section{Study Population And Sample}

The study population was composed of all maternity staff members in 2010 and 2014. They first selected facilities and then interviewed all staff members in each of them. In both the 2010 and 2014 surveys, the questionnaire was administered to all those who were present in the maternity ward on the day of the visit.

Supporting staff who were not involved in deliveries were excluded. Gynecologists, pediatricians, medical residents and nursing college trainees, as well as medical students, were also not considered in the analysis sample.

The first round (2010) was conducted from May to June 2010 in 1982 HCs across the country. However, in total, 1626 performed at least one delivery in the 12 months prior to the visit. The EmONC needs assessment and services review were performed in those facilities.

In 2014, the final analysis sample consisted of 789 HCs, including the following: all hospitals (112); HCs located in municipalities, main towns or villages (312); and 342 others randomly selected among $1860 \mathrm{HCs}$, including 1554 public and 306 private HCs and clinics. This sample was proportionally allocated to the regions on the basis of the number of facilities by the end of 2012, as reported by the Ministry of Health.

For the purposes of this study, we extracted data on facilities' characteristics and all health workers' interviews in facilities that have been repeatedly surveyed (2010 and 2014). However, we excluded the facilities that were upgraded between 2010 and 2014.

\section{Variables}

We extracted data on facilities' general information, such as type of facility (HC or hospital), location (urban or rural), administrative region and managing authority (public or private).

At the individual level, we extracted data on staff qualifications (midwife, assistant midwife and nurse) and their training background (yes or no) on each of the following training topics: focused antenatal care (FANC), partogram utilization, active management of the third stage of labor (AMTSL), manual removal of the placenta, parenteral administration of magnesium sulfate, suturing cervical tears, suturing vaginal tears, use of ventouse and forceps, manual vacuum aspiration (MVA) and prevention of mother-to-child transmission (PMTCT) of HIV.

Regarding variables for staff knowledge, Table 1 presents the thematic questions along with the expected answers. For each question, each mentioned item accounted for one point; otherwise, the item accounted for 0 . For each health worker, we first computed an individual-level score (percentage) of knowledge by topic by dividing the number of mentioned items times one hundred by the total number of expected items. We then calculated the health facility level mean percentage of knowledge for each topic in 2010 and 2014 because the paired statistical units in this study were the facilities, not individual staff members. Finally, we categorized the facility level score into 3 groups: $0 \%$ to $49.99 \%$ as a low level of knowledge, $50 \%$ to $74.99 \%$ as a medium level of knowledge, and equal to or greater than $75 \%$ as a high level of knowledge.

\section{Statistical Analysis}

For each topic, we computed the differences in percentages (percentages for 2014 minus those for 2010) and 


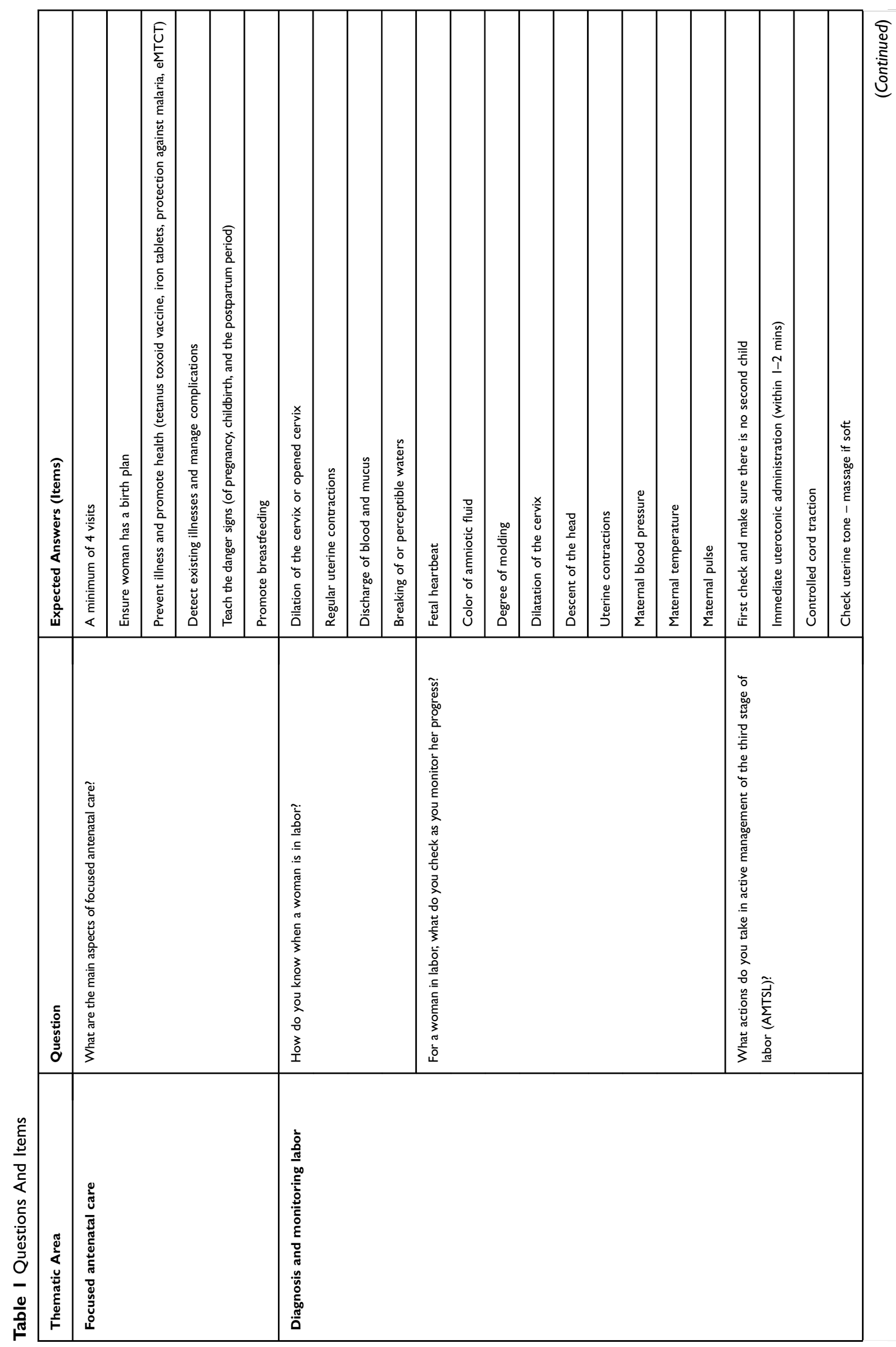




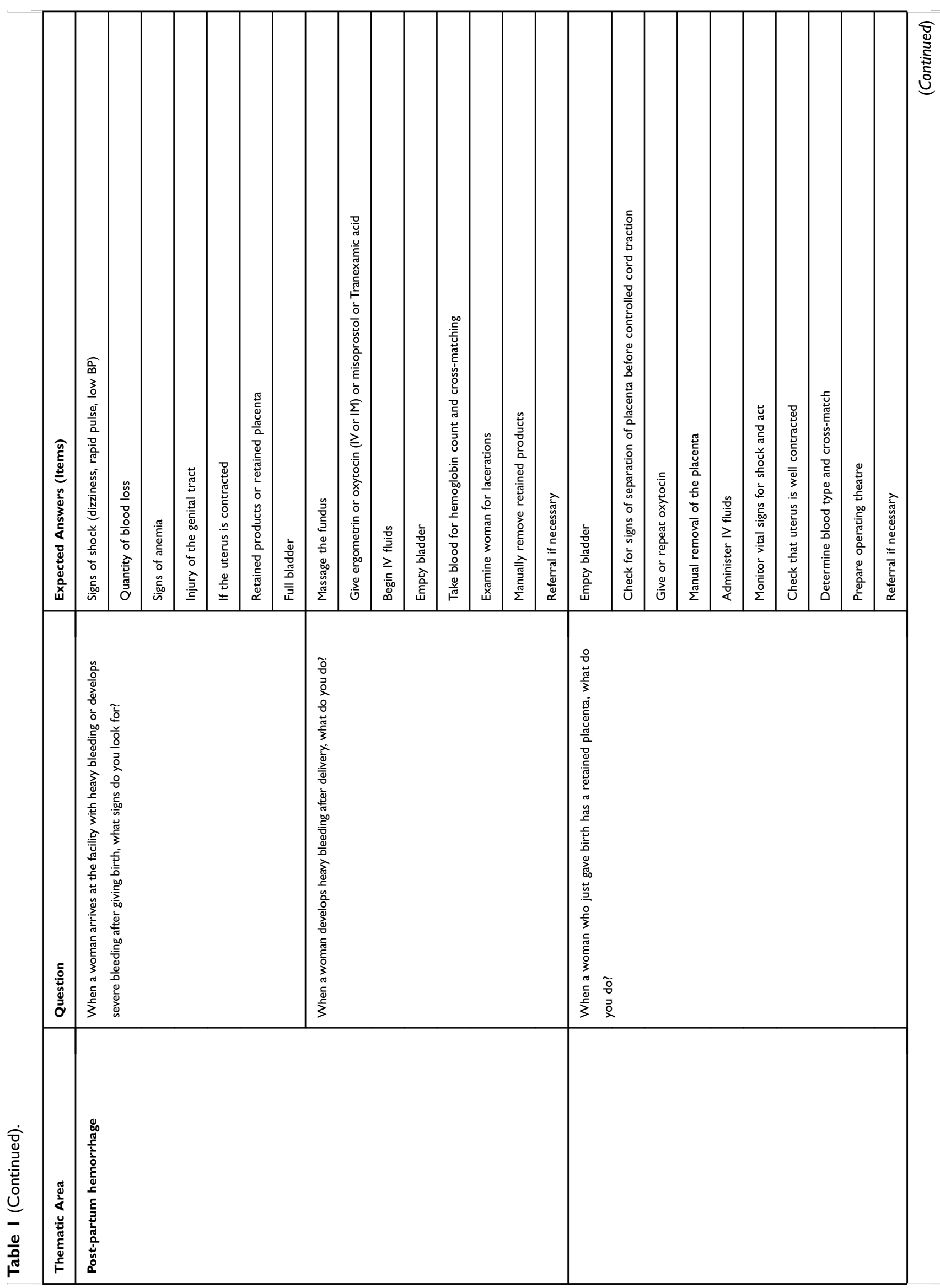




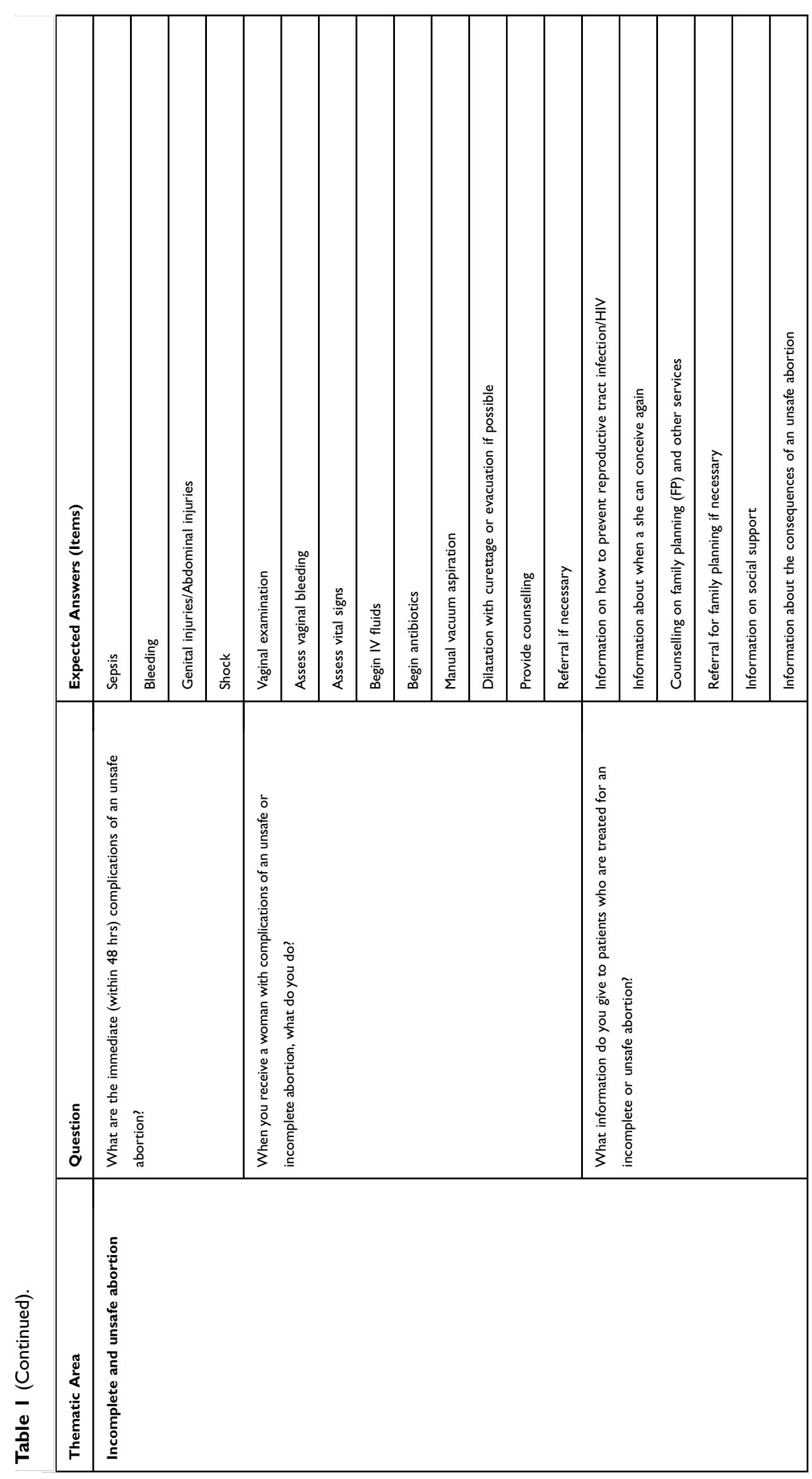


used McNemar's test for dependent samples to compare the proportions with a $5 \%$ significance level. Data extraction and analysis were performed using Stata 15.1.

\section{Ethical Considerations}

Field workers were trained prior to the assessments to conduct confidential interviews only with the interviewee. No one else was allowed in the room during the interviews. This procedure was to protect participants from embarrassment or any other harm if they showed a low level of knowledge.

The questionnaire on staff knowledge was anonymous, as no individual-level identification was needed for the assessments. During both assessments, participants provided written informed consent before the start of the interview. The protocols were submitted to and approved by Burkina Faso Health Research Ethics Committee in 2010 and 2014.

\section{Results}

\section{Characteristics Of Health Facilities}

Of the 789 facilities surveyed in 2014, 736 (93.3\%) were also visited in 2010 and were therefore included in our analysis. Most of them were primary healthcare centers $(87.2 \%)$. One-fifth $(21.6 \%)$ were located in urban areas, and $95.7 \%$ were managed by the government. The characteristics of the facilities are presented in Table 2 .

Table 2 Characteristics Of The Facilities

\begin{tabular}{|l|l|l|}
\hline Variable & $\mathbf{n} \%$ & \\
\hline Type of facility & & 0.3 \\
University teaching hospital & 2 & 1.2 \\
Regional hospital & 9 & 5.7 \\
District hospital & 42 & 2.3 \\
Medical center* & 17 & 87.2 \\
Health center & 642 & 3.3 \\
Other (maternities) & 24 & \\
\hline Location & & 21.6 \\
Urban & 159 & 78.4 \\
Rural & 577 & \\
\hline Managing authority & & 95.7 \\
Public & 697 & 3.4 \\
Private & 25 & 1.9 \\
NGO/Faith-based & 14 & 100.0 \\
\hline Total & $\mathbf{7 3 6}$ & \\
\hline
\end{tabular}

Note: *Health center with at least one general practitioner, a laboratory for basic tests.

\section{Characteristics Of Health Workers}

In 2010, and 2014, 861 and 979 maternity staff members were surveyed, respectively. In 2010, 21.6\% $(n=187)$ of them were midwives, whereas in 2014 , this category accounted for $39.0 \%(n=382)$ of the sample (Table 3$)$.

\section{Staff Training}

From 2010 to 2014, the proportion of staff who received training on FANC and AMSTL increased by $15.8 \%$ [from $70.4 \%(\mathrm{n}=607)$ to $86.2 \%(\mathrm{n}=843)$ ] and $14.7 \%$ [from $74.7 \%$ $(\mathrm{n}=644)$ to $89.4 \%(\mathrm{n}=884)]$, respectively. Likewise, the percentage of those who were trained in parenteral administration of magnesium sulfate $[32.1 \%(\mathrm{n}=277)$ and $50.9 \%$ ( $\mathrm{n}=497)$ ] and MVA (23.9\% and 40.4\%) increased as well. There was no significant variation in the proportion of staff who received training on manual placenta removal $[82.4 \%$ $(\mathrm{n}=710)$ to $83.4 \%(\mathrm{n}=815)]$ (Table 4$)$.

\section{Focused Antenatal Care}

Overall, the proportion of the facilities in which health workers had a low level of knowledge on FANC significantly decreased from $59.5 \%(\mathrm{n}=420)$ to $39.3 \%(\mathrm{n}=280)$. The proportion of those with a high level of knowledge increased by 3.3\% $(\mathrm{p}<0.001)$. In HCs, the proportion of facilities with a low level of knowledge decreased by $21.1 \%$, whereas those with a high level increased by $3.5 \%(\mathrm{p}<0.001)$, as shown in Table 5 . The variation was not significant in hospitals $(\mathrm{p}=0.304)$.

\section{Diagnosing And Monitoring Labor}

The proportion of facilities in which staff members had a low level of knowledge on the parameters of the start of labor, monitoring of labor progress and AMSTL declined by $10.1 \%$ [from $34.7 \% \quad(n=246)$ to $24.6 \% \quad(n=175)$, $\mathrm{p}<0.001$ ], $18.3 \%$ [from $28.9 \%(\mathrm{n}=205)$ to $10.5 \%(\mathrm{n}=75)$, $\mathrm{p}<0.001$ ] and $29.5 \%$ [from $66.9 \%(\mathrm{n}=471)$ to $37.4 \%$ $(\mathrm{n}=266), \mathrm{p}<0.001]$, respectively (Table 5$)$.

\section{Postpartum Hemorrhage: Diagnosis And Management}

Regarding the screening of women with heavy bleeding during the postpartum period, in 2010, $76.9 \%(n=545)$ of the facilities had staff members with a low level of knowledge, and that proportion declined by $5.7 \%(\mathrm{p}<0.05)$. However, there was no significant variation in hospitals $(\mathrm{p}=0.304)$.

With regard to the management of postpartum bleeding, in HCs, there was a $10.1 \%$ [62.1\% $(\mathrm{n}=403)$ in 2010 and $52.0 \%(n=338)$ in 2014] decrease in the proportion of 
Table 3 Percentage Of Care Providers By Qualification In 2010 And 2014

\begin{tabular}{|l|l|l|l|l|}
\hline \multirow{2}{*}{ Category } & \multicolumn{2}{|l|}{$\mathbf{2 0 1 0}$} & \multicolumn{2}{l|}{$\mathbf{2 0 1 4}$} \\
\cline { 2 - 5 } & $\mathbf{n}$ & $\%$ & $\mathbf{n}$ & $\%$ \\
\hline Midwife & 187 & 21.6 & 382 & 39.0 \\
Assistant midwife & 478 & 55.3 & 461 & \\
Nurse & 110 & 12.7 & 70 & \\
Other & 90 & 10.4 & 66 & \\
Total & 865 & 100.0 & 979 & 100.0 \\
\hline
\end{tabular}

Table 4 Percentage Of Staff Trained In 2010 And 2014 On Selected Topics In Burkina Faso

\begin{tabular}{|l|l|l|l|l|l|}
\hline \multirow{2}{*}{ Topics } & \multicolumn{2}{l|}{$\mathbf{2 0 1 0}$} & \multicolumn{2}{l|}{$\mathbf{2 0 1 4}$} & Difference (\%) \\
\cline { 2 - 6 } & $\mathbf{n}$ & \% & $\mathbf{n}$ & \% & (\%2010 - \%2014) \\
\hline Focused antenatal care & 607 & 70.4 & 843 & 86.2 & 15.8 \\
Partogram & 713 & 82.7 & 884 & 90.4 & 7.7 \\
AMTSL & 644 & 74.7 & 874 & 89.4 & 14.7 \\
Manual removal of the placenta & 710 & 82.4 & 815 & 83.4 & 1.1 \\
Parenteral administration of Magnesium sulfate & 277 & 32.1 & 497 & 50.9 & 18.7 \\
Suture of vaginal tears & 397 & 46.1 & 541 & 55.4 & 9.3 \\
Suture of cervical tears & 112 & 13.0 & 203 & 20.8 & 7.8 \\
Use of ventouse & 135 & 15.7 & 287 & 29.4 & 13.7 \\
Use of forceps & 107 & 12.4 & 195 & 20.0 & 7.6 \\
Vacuum manual aspiration & 206 & 23.9 & 395 & 40.4 & 16.5 \\
Prevention of mother-to-child transmission of HIV & 658 & 76.4 & 829 & 84.8 & 8.4 \\
Neonatal resuscitation & 421 & 48.8 & 616 & 63.1 & 14.2 \\
Integrated management of childhood illness & 102 & 11.8 & 177 & 18.1 & 6.3 \\
\hline
\end{tabular}

facilities with a low level of knowledge $(\mathrm{p}<0.001)$. Meanwhile, the proportion of those with medium and high levels of knowledge increased by $8.3 \%$ and $1.8 \%$, respectively. There was no significant variation in hospitals $(p=0.206)$ (Table 5).

The proportion of facilities with a low level of knowledge of the management of retained placenta did not vary over the 5-year period: from $89.3 \%(n=631)$ to $88.3 \%$ $(n=628), \quad(p=0.553)$ in general; from 90.2\% $(n=55)$ to $83.9(\mathrm{n}=52)(\mathrm{p}=0.317)$ in hospitals; and from $89.2 \%$ $(\mathrm{n}=576)$ to $88.8 \%(\mathrm{n}=576)(\mathrm{p}=0.787)$ in HCs (Table 5).

\section{Incomplete And Unsafe Abortion}

Regarding incomplete or unsafe abortion, we analyzed knowledge of immediate complications, postabortion care and main information to provide to a patient admitted for incomplete or unsafe abortion. There was no significant variation among hospitals staff members in knowledge of immediate complications $(\mathrm{p}<0.162)$, postabortion care $(\mathrm{p}<0.317)$ and main information to provide to a patient admitted for incomplete or unsafe abortion $(p<0.304)$. In contrast, in HCs, there was a significant improvement, except for the information to provide in cases of unsafe abortion $(\mathrm{p}=0.167)$. The percentage of facilities with a low level of knowledge decreased by $8.1 \%$ [from $56.7 \%$ $(n=367)$ to $46.8 \%(n=316), p<0.001]$ for immediate complications and $9.7 \%$ [from $81.7 \% \quad(n=528)$ to $72.0 \%$ $(\mathrm{n}=468), \mathrm{p}<0.001]$ for postabortion care (Table 5).

\section{Discussion}

\section{Staff Qualification And Training}

The proportion of midwives among the nonmedical staff of maternity wards has increased from $21.6 \%$ in 2010 to $39 \%$ in 2014 . In addition, our study showed an increasing proportion of staff members who received training on obstetric care topics. These results are in line with the objective of the country strategic plan for maternal and child health adopted in 2006, which called for the availability of quality EmONC, including constant antenatal care. This was supposed to be achieved through the strengthening of the health system and human resource development. $^{27}$ Considering the importance of midwives 


\begin{tabular}{|c|c|c|c|c|c|c|c|c|c|c|c|}
\hline \multirow{4}{*}{ 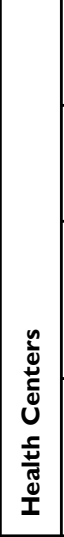 } & a & $\bar{o}$ & হò & 호 & $\bar{o}$ & $\stackrel{n}{\stackrel{u}{\dot{v}}}$ & so & $\begin{array}{l}\stackrel{\infty}{\infty} \\
\stackrel{0}{0}\end{array}$ & 늠 & o̊̀ & $\frac{\hat{0}}{0}$ \\
\hline & 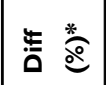 & $\stackrel{\sim}{T} \stackrel{g}{\stackrel{m}{\Gamma}} \underset{m}{m}$ & $\stackrel{t}{\circ} \circ \stackrel{m}{a}$ & 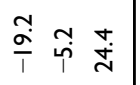 & बें & $\hat{p} \hat{m} \stackrel{i}{i}$ & $\bar{\varphi} \underset{\infty}{\infty} \underset{\infty}{\infty} \stackrel{\infty}{=}$ & $\stackrel{t}{0}$ ำ & 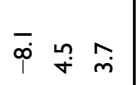 & $\hat{i} \stackrel{\infty}{\sim} \stackrel{0}{\sim}$ & $\stackrel{0}{\ddot{i}} \underset{+}{+} \stackrel{\infty}{\stackrel{\infty}{i}}$ \\
\hline & 芛 & 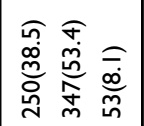 & 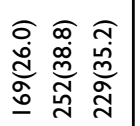 & 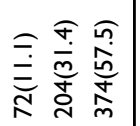 & 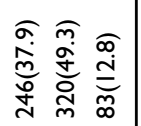 & 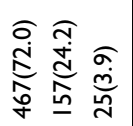 & 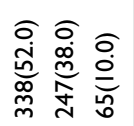 & 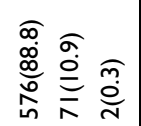 & 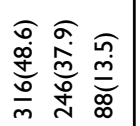 & 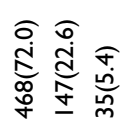 & 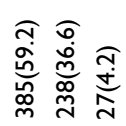 \\
\hline & 융 & 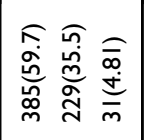 & 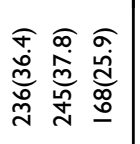 & 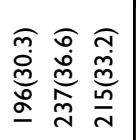 & 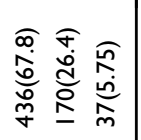 & 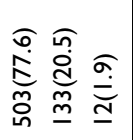 & 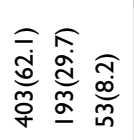 & 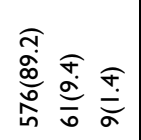 & 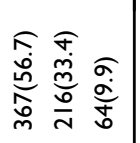 & 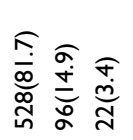 & 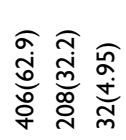 \\
\hline \multirow[b]{4}{*}{$\begin{array}{l}\frac{n}{N} \\
\frac{\Delta}{0} \\
\frac{\hat{n}}{1} \\
\text { I }\end{array}$} & a & $\begin{array}{l}\text { tr } \\
0 \\
0 \\
0\end{array}$ & 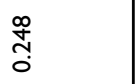 & $\begin{array}{l}\infty \\
\stackrel{\infty}{0} \\
0 \\
0\end{array}$ & $\begin{array}{l}\text { Lo } \\
\text { o. } \\
\text { v }\end{array}$ & 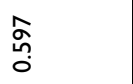 & $\underset{\tilde{N}}{\stackrel{0}{0}}$ & $\frac{n}{m}$ & $\frac{\pi}{0}$ & $\frac{n}{m}$ & $\underset{\substack{0 \\
0}}{0}$ \\
\hline & 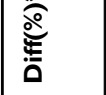 & 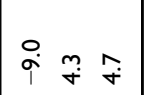 & $\stackrel{\wedge}{\varphi} \stackrel{0}{i} \stackrel{\infty}{\Gamma}$ & $\hat{i} \bar{i} \quad \stackrel{\infty}{\dot{\varphi}}$ & 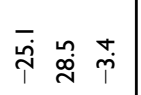 & 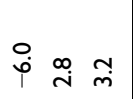 & 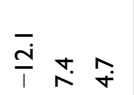 & 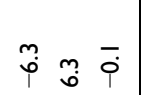 & 定芒道 & 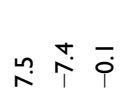 & $\stackrel{+}{=} \stackrel{\sim}{\underline{\underline{T}}} \stackrel{\infty}{T}$ \\
\hline & 茟 & 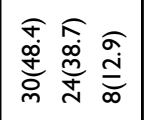 & 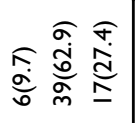 & 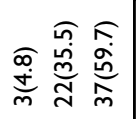 & 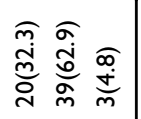 & 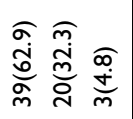 & 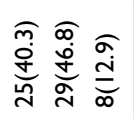 & 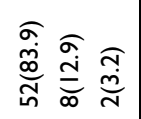 & 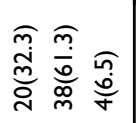 & 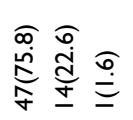 & 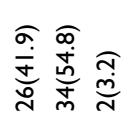 \\
\hline & 응 & 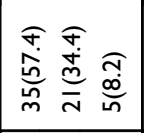 & 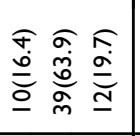 & 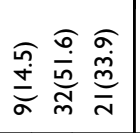 & 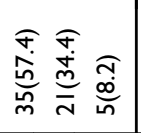 & 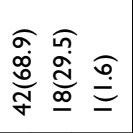 & 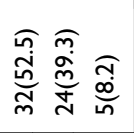 & 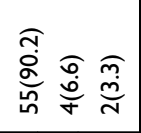 & 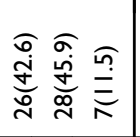 & 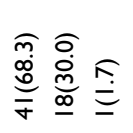 & 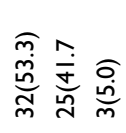 \\
\hline \multirow{4}{*}{ 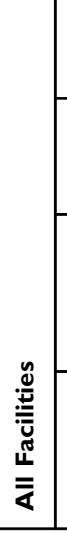 } & a & $\bar{o}$ & হ⿺ & sọ & sọ & $\begin{array}{l}\text { Lo } \\
\text { v }\end{array}$ & চे & 崅 & $\overline{0}$ & চे & $\frac{\tilde{\sigma}}{0}$ \\
\hline & 纪 & 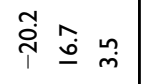 & $\overline{0}$ 웅 & 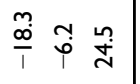 & 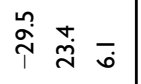 & $\hat{\varphi} \stackrel{\sim}{\dot{m}} \bar{i}$ & $\stackrel{m}{0}_{i}^{\infty} \underset{i}{i}$ & $\stackrel{a}{i} \underline{\underline{i}} \frac{0}{i}$ & 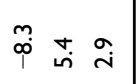 & 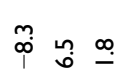 & $\stackrel{m}{\dot{y}}$ ถู \\
\hline & 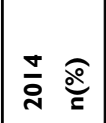 & 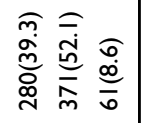 & 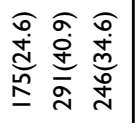 & 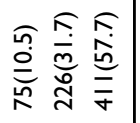 & 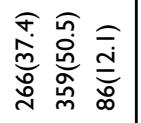 & 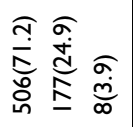 & 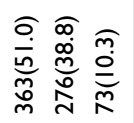 & 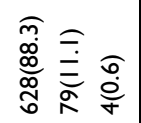 & 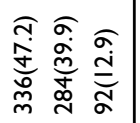 & 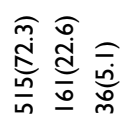 & 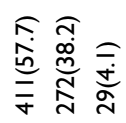 \\
\hline & 응 & 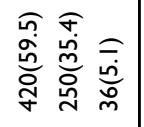 & 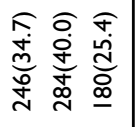 & 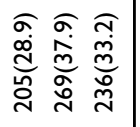 & 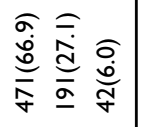 & 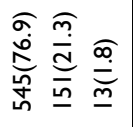 & 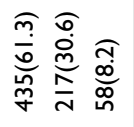 & 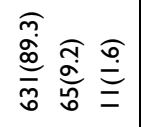 & 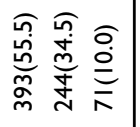 & 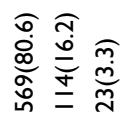 & 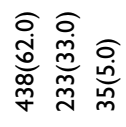 \\
\hline & & 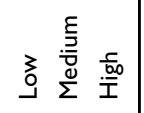 & 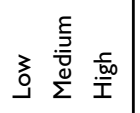 & 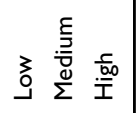 & 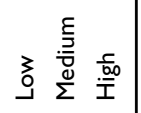 & 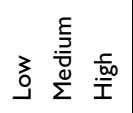 & 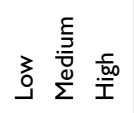 & 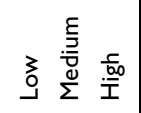 & 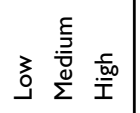 & 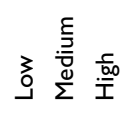 & 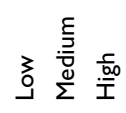 \\
\hline 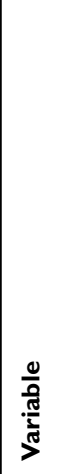 & & 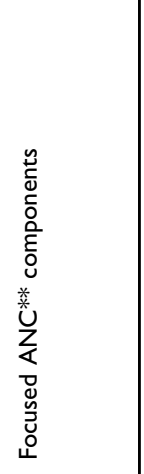 & 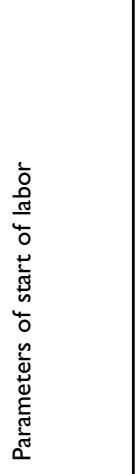 & 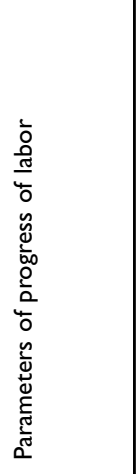 & 举 & 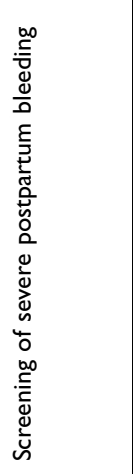 & 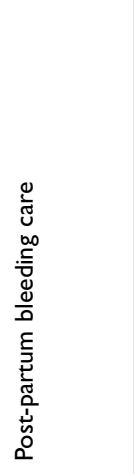 & 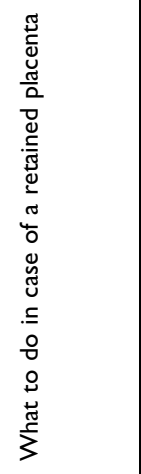 & 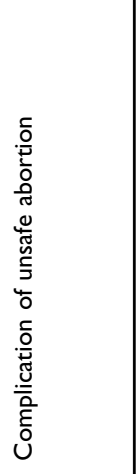 & 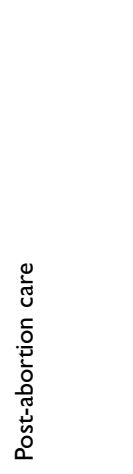 & 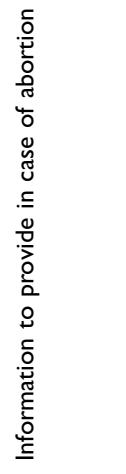 \\
\hline
\end{tabular}


in delivering maternity care services, ${ }^{28}$ the country aimed to achieve better availability of midwives and nurses with midwives' competencies in its maternity wards. The total number of midwives in Burkina Faso increased from 1057 in $2010^{29}$ to 1591 in $2014 .{ }^{24}$ Previous studies reported that midwives have competencies and skills close to those of physicians in basic obstetric care. ${ }^{30,31}$ Therefore, this increase in the proportion and training reflects the effort and progress towards better service delivery.

During the last years of the era of MDG 5, all countries were pushing to reach their targets. However, most programs have not evaluated the effect of their capacity-building components on the level of knowledge of maternity staff. Training has become a routine activity in the Burkina Faso health system and is rarely evaluated. This is crucial because there are doubts raised with respect to the efficacy of in-service training and supervision programs in sub-Saharan Africa. ${ }^{32}$ In a study involving seven subSaharan countries, Leslie et al analyzed the association between in-service training and supervision and quality of antenatal and sick child care. Their results showed that inservice training and supervision, as delivered in those countries, are insufficient to ensure delivery of good quality service. ${ }^{32}$ Although staff members are increasingly available and trained, evaluation could add value to the quality of services. In particular, the results of progress monitoring and evaluation should be routinely shared with policymakers and program managers to improve capacitystrengthening activities.

\section{Improved Knowledge In Health Centers But Not In Hospitals}

This study showed that staff knowledge on how to deliver maternity care of good quality has increased overall. The improvement was more significant in HCs than in hospitals. There is no previous study on this topic for comparison. Nevertheless, our hypothesis was that this may be a consequence of the fact that basic obstetric care-related capacity-strengthening programs targeted health centers more than hospitals. Theoretically, HCs have lower-level staff; hence, they need more training for some of the services. Hospitals, as reference centers, are often not the first target for training sessions that do not involve caesarean section or other obstetric surgery. Doctors and midwives are supposed to be available in those facilities. But in most of the hospitals in Burkina Faso, doctors do not remain on duty constantly. Often, the available staff members in hospitals (except in teaching hospitals) for maternity care tend to be the same as those in HCs. They usually call physicians only in cases of emergency. ${ }^{26}$

As in many sub-Saharan African countries, the healthcare-seeking path is not strongly established. Uncomplicated cases can enter the system through both HCs and hospitals. Ideally, hospitals are expected to receive the most complicated cases and referrals. ${ }^{26,33}$ Since this cannot be enforced in most developing countries, considering hospital maternity staff in capacity-strengthening programs, even for basic services, can improve quality of care. This should also include the equipment required for those services. Even in HCs, although the proportion of individuals with low level of knowledge decreased, the increase in the proportion of individuals with high levels of knowledge, namely, those who responded with more than $75 \%$ of the correct answers, was not as important. The main change was noticed in the proportion of those with medium level of knowledge. These results suggest that between 2010 and 2014, progress was made in levels of knowledge, but much remains to be accomplished to bring staff knowledge to the highest level.

\section{Knowledge Of The Management Of Retained Placenta Did Not Improve}

The proportion of facilities where staff had a low level of knowledge of the management of retained placenta remained very high $(89.2 \%$ in 2010 and $88.3 \%$ in 2014) throughout the study period. The proportion of those with high level of knowledge even decreased by $1 \%$ (Table 5 ). These results can be explained by the fact that health workers focus on the manual removal of the placenta as the main course of action in managing retained placenta. This procedure was mentioned by $79 \%$ of health workers in 2010 and $82.5 \%$ in 2014 (data not reported). They rarely mention additional procedures, such as taking blood samples for tests and preparing the theatre. In this study, we included many more items in the assessment of knowledge of this topic. Therefore, this result may not reflect actual practice.

\section{Strength And Limitations}

This study is the first to analyze the progress in staff knowledge of maternal health services in Burkina Faso. In addition to drawing the attention of policymakers toward key topics to consider in capacity strengthening for maternity health workers, this study can serve as a baseline for monitoring and evaluating the development 
of human resources in maternity wards. Furthermore, our findings are generalizable at the national level in hospitals and primary health centers when considered separately. These results can also be useful for other sub-Saharan African countries in which maternity care is mostly provided by nurses due to the shortage of medical doctors and midwives.

In this study, we had to consider some weaknesses and limitations due to the fact that the national needs assessments only considered care providers' spontaneous responses. Health workers were not observed in actual practice, and they did not use vignettes. ${ }^{34}$ We also did not assess the availability of the equipment required for these services. These findings should therefore be considered as an assessment of their theoretical knowledge, which may differ from their actual practice. However, because theoretical knowledge is key to good practice and quality, this study provides an opportunity to appraise how well frontline maternity care providers can perform and make progress.

The level of knowledge could have been underestimated, given that facilities have no standard operating procedures on the study variables. Although there is a national protocol on how to perform these tasks, participants from different backgrounds can consider some items and not others, and therefore underestimate the proportion or hinder the comparability among groups.

\section{Conclusion}

From 2010 to 2014 , there was a significant increase in the proportion of midwives in maternity wards. Staff knowledge has also improved, but there is still room for improvement, as most of the facilities reported a medium level of staff knowledge. This finding suggests a critical appraisal of the current approaches of training and capacity building. Hospitals seem to have been left behind, as no significant variation was identified in the study period. Capacity-strengthening programs should take hospitals maternity staff members into account, because in Burkina Faso, women seek basic EmONC in both hospitals and HCs. Therefore, hospitals should also be ready to offer both basic and specialized maternity care. In addition, our results suggest that training and other capacitybuilding approaches should be revised to ensure that those who attend the training obtain good posttraining supervision and monitoring to reinforce the knowledge they acquired.

\section{Acknowledgments}

We are grateful to the AMDD technical advisors who contributed to the planning and implementation of the survey in 2010. We thank Vuyokazi Jezile and Eliane Kima, whose contributions to proofreading and editing this paper were valuable.

\section{Author Contributions}

All authors contributed to data analysis, drafting or revising the article, gave final approval of the version to be published, and agree to be accountable for all aspects of the work.

\section{Funding}

The UNFPA funded both rounds of the EmONC national needs assessments (2010 and 2014). The WHO contributed to the funding of the assessment in 2010.

\section{Disclosure}

Dr Adama Baguiya report grants from UNFPA, during the conduct of Burkina Faso 2010 and 2014 Emergency Obstetric and Newborn Care needs assessment. The authors report no other conflicts of interest in this work.

\section{References}

1. Alkema L, Chou D, Hogan D, et al. Global, regional, and national levels and trends in maternal mortality between 1990 and 2015, with scenario-based projections to 2030: a systematic analysis by the UN Maternal Mortality Estimation Inter-Agency Group. Lancet. 2016;387 (10017):462-474. doi:10.1016/S0140-6736(15)00838-7

2. United Nations. Sustainable development goals. U N Sustain Dev' 2016. Available from: http://www.un.org/sustainabledevelopment/sus tainable-development-goals/. Accessed October 27, 2016.

3. UN Inter-Agency Group for, Child Mortality Estimation. Levels and Trends in Child Mortality Report 2015. New York; 2015:36.

4. World Health Organization, UNICEF, United Nations, Department of Economic and Social Affairs, Population Division, World Bank. Trends in maternal mortality: 1990 to 2015: estimates by WHO, UNICEF, UNFPA, World Bank Group and the United Nations Population Division; 2015. Available from: http://www.who.int/repro ductivehealth/publications/monitoring/maternal-mortality-2015/en/. Accessed January 24, 2017.

5. United Nations. The Millennium Development Goals Report 2015. New York; 2015:75. Available from: http://www.un.org/millenniumgoals/ 2015_MDG_Report/pdf/MDG\%202015\%20rev\%20(July\%201).pdf.

6. Tunçalp Ö, Were W, MacLennan C, et al. Quality of care for pregnant women and newborns-the WHO vision. BJOG Int J Obstet Gynaecol. 2015;122(8):1045-1049. doi:10.1111/1471-0528.13451

7. Sharma G, Mathai M, Dickson KE, et al. Quality care during labour and birth: a multi-country analysis of health system bottlenecks and potential solutions. BMC Pregnancy Childbirth. 2015;15(Suppl 2):S2. doi:10.1186/1471-2393-15-S2-S2

8. Graham WJ, Varghese B. Quality, quality, quality: gaps in the continuum of care. Lancet Lond Engl. 2012;379(9811):e5-e6. doi:10.10 16/S0140-6736(10)62267-2 
9. Kruk ME, Larson E, Twum-Danso NAY. Time for a quality revolution in global health. Lancet Glob Health. 2016;4(9):e594-e596. doi:10.1016/S2214-109X(16)30131-0

10. Sharma J, Leslie HH, Kundu F, Kruk ME. Poor quality for poor women? Inequities in the quality of antenatal and delivery care in Kenya. PLoS One. 2017;12(1):e171236. DeWan AT, ed. doi:10.1371/journal.pone. 0171236

11. Bacci A. Quality of maternal and neonatal care in Central Asia and Europe-lessons learnt. BJOG Int J Obstet Gynaecol. 2014;121 (S4):11-14. doi:10.1111/1471-0528.12819

12. Powell-Jackson T, Mazumdar S, Mills A. Financial incentives in health: new evidence from India's Janani Suraksha Yojana. $J$ Health Econ. 2015;43:154-169. doi:10.1016/j.jhealeco.2015.07.001

13. Ng M, Misra A, Diwan V, Agnani M, Levin-Rector A, Costa AD. An assessment of the impact of the JSY cash transfer program on maternal mortality reduction in Madhya Pradesh, India. Glob Health Action. 2014;7. doi:10.3402/gha.v7.24939

14. Kyei-Nimakoh M, Carolan-Olah M, McCann TV. Access barriers to obstetric care at health facilities in sub-Saharan Africa-a systematic review. Syst Rev. 2017;6(1):110. doi:10.1186/s13643-017-0503-x

15. Say L, Chou D, Gemmill A, et al. Global causes of maternal death: a WHO systematic analysis. Lancet Glob Health. 2014;2(6):323-333. doi:10.1016/S2214-109X(14)70227-X

16. Bhutta ZA, Das JK, Bahl R, et al. Can available interventions end preventable deaths in mothers, newborn babies, and stillbirths, and at what cost? Lancet. 2014;384(9940):347-370. doi:10.1016/S01406736(14)60792-3

17. INSD. Enquête Multisectorielle Continue (EMC) 2014. Santé Générale et Santé de La Reproduction. Ouagadougou: INSD; 2015:68.

18. The Partnership for Maternal, Newborn \& Child Health. PMNCH 2014 Progress Report: Moving into 2015 and Beyond. Geneva, Switzerland: WHO; 2015:57.

19. Ridde V, Richard F, Bicaba A, Queuille L, Conombo G. The national subsidy for deliveries and emergency obstetric care in Burkina Faso. Health Policy Plan. 2011;26(suppl_2):ii30-ii40. doi:10.1093/heapol/ czr060

20. WHO. H4+ Works Together Pro-Actively with Partners at Country, Regional and Global Level. WHO. Available from: http://www.who.int/ life-course/partners/h4/h4-plus-partners/en/. Accessed August 29, 2018.

21. RBF Health. Performance-Based Financing (PBF) Burkina Faso. Available from: https://www.rbfhealth.org/rbfhealth/country/burkinafaso. Accessed August 29, 2018.

22. Kim YM, Ansari N, Kols A, et al. Assessing the capacity for newborn resuscitation and factors associated with providers' knowledge and skills: a cross-sectional study in Afghanistan. BMC Pediatr. 2013;13:140. doi:10.1186/1471-2431-13-140
23. Kouanda S, Ouédraogo AM, Ouédraogo GH, Sanon D, Belemviré S, Ouédraogo L. Emergency obstetric and neonatal care needs assessment: results of the 2010 and 2014 surveys in Burkina Faso. Int J Gynecol Obstet. 2016;135(S1):S11-S15. doi:10.1016/ j.ijgo.2016.08.008

24. Ministry of Health, Burkina Faso. Annual Health Statistics 2014. Ouagadougou: Ministry of Health of Burkina Faso; 2015:330.

25. Ministry of Health of Burkina Faso. Annual Health Statistics 2015. Ouagadougou: Ministry of Health of Burkina Faso; 2016:342.

26. Research Institute of Health Sciences (IRSS) and the Ministry of Health Burkina Faso. Emergency Obstetric and Newborn Care (EmONC) Needs Assessment and Reproductive Health Care Mapping in Burkina Faso 2010. Ouagadougou: Ministry of Health of Burkina Faso; 2011:352.

27. Ministry of Health, Burkina Faso. Maternal and Neonatal Mortality Reduction Acceleration Plan (Roadmap). Ouagadougou: Ministry of Health of Burkina Faso; 2006:45.

28. Horton R, Astudillo O. The power of midwifery. Lancet. 2014;384 (9948):1075-1076. doi:10.1016/S0140-6736(14)60855-2

29. Ministry of Health of Burkina Faso. Annual Health Statistics 2010. Ouagadougou: Ministry of Health of Burkina Faso; 2011:204.

30. Traoré M, Arsenault C, Schoemaker-Marcotte C, et al. Obstetric competence among primary healthcare workers in Mali. Int $J$ Gynecol Obstet. 2014;126(1):50-55. doi:10.1016/j.ijgo.2014. 01.012

31. Kim Y-M, Mungia J, Faqir M, Ansari N, Evans C. Patterns in training, knowledge, and performance of skilled birth attendants providing emergency obstetric and newborn care in Afghanistan. Int J Gynecol Obstet. 2012;119(2):125-129. doi:10.1016/j.ijgo.2012.05.030

32. Leslie HH, Gage A, Nsona H, Hirschhorn LR, Kruk ME. Training and supervision did not meaningfully improve quality of care for pregnant women or sick children in subSaharan Africa. Health Aff (Millwood). 2016;35(9):1716-1724. doi:10.1377/hlthaff.2016.0261

33. Meda IB, Millogo T, Baguiya A, Nikiema L Ouédraogo/, Coulibaly A, Kouanda $\mathrm{S}$. Rate of and factors associated with indications for cesarean deliveries: results of a national review in Burkina Faso. Int J Gynecol Obstet. 2016;135(S1):S51-S57. doi:10.1016/j.ijgo.20 16.08.010

34. Lohela TJ, Nesbitt RC, Manu A, et al. Competence of health workers in emergency obstetric care: an assessment using clinical vignettes in Brong Ahafo region, Ghana. BMJ Open. 2016;6(6):e010963. doi:10.1136/bmjopen-2015-010963

\section{Publish your work in this journal}

The International Journal of Women's Health is an international, peerreviewed open-access journal publishing original research, reports, editorials, reviews and commentaries on all aspects of women's healthcare including gynecology, obstetrics, and breast cancer. The manuscript management system is completely online and includes a very quick and fair peer-review system, which is all easy to use. Visit http://www.dovepress.com/testimonials.php to read real quotes from published authors. 